PREPRINT:

\title{
WHISTLER WAVES \\ OBSERVED UPSTREAM FROM \\ COLLISIONLESS SHOCKS
}

D. FAIRFIELD

(NASA-TM-X-66245) WHISTLER WAVES OBSERVED
UPSTREAM FROM COLLISIONLESS SHOCKS (NASA)
$61 \mathrm{pHC} \$ 5.25$
CSCL O4A G3/13 Unclas

GSFC GODDARD SPACE FLIGHT CENTER

\section{GREENBELT, MARYLAND}




\section{WHISTLER WAVES OBSERVED UPSTREAM FROM COLLISONLESS SHOCKS}

D.H. Fairfield

May 1973 
Abstract

Waves in the frequency range $0.5-4 \mathrm{hz}$ have been studied in the region upstream of the earths bow shock using data from the fluxgate magnetic field experiment on IMP-6. Such waves are invariably detected adjacent to the shock, persisting upstream for intervals often less than a minute but occasionally of the order of many hours. Analysis of 150 examples of these waves during a three month interval indicates that amplitudes are generally less than 1 or 2 gammas $(\Delta B / B \simeq 0.2)$ and propagation directions generally make angles of between $20^{\circ}$ and $40^{\circ}$ with the field direction. The waves as measured in the spacecraft frame of reference are either left or right hand polarized with respect to the average field direction. The left handed waves generally have lower frequencies than the right handed waves and the left handed frequencies never exceed $2.5 \mathrm{hz}$. The measured sense of polarization is found to depend on the propagation direction (or alternatively the field direction) relative to the solar wind direction. Propagation-solar wind angles greater than approximately $40^{\circ}$ (or field angles greater than $55^{\circ}$ ) correspond to right hand polarization and smaller angles correspond to left handed polarization. It is concluded that the observed waves are 
right handed waves in the plasma frame of reference with wavelengths of approximately $100 \mathrm{~km}$ propagating upstream in the whistler mode. Doppler shifting reduces the observed frequencies in the spacecraft frame and reverses the observed polarization for those waves propagating more directly upstream. Similar waves are seen ahead of most interplanetary shocks. 
I. Introduction

Large amplitude quasi-sinusoidal waves upstream from the earth's bow shock appear to fall naturally into two frequency ranges of approximatelv...01-.05 $\mathrm{Hz}$ and $0.5-4 . \mathrm{hz} .$. The lower frequency waves have been studied by Fairfield (1969) and Greenstadt et al. (1970b) and their properties are fairly well-known. They are primarily transverse waves of several gammas amplitude and they exhibit left hand polarization in the spacecraft frame of reference. The waves are associated with the bow shock since they are only observed on interplanetary field lines that connect with the shock (Fairfield, 1969). Furthermore these waves appear to be instrumental in determining the observed character of the bow shock (Greenstadt et al., 1970a; 1970c; Greenstadt, 1972). It has been suggested that these waves are locally generated in the solar wind by protons moving upstream from the shock (Fairfield, 1969; Barnes 1970; Greenstadt et al. 1970b).

Characteristics of the $0.5-4$. hz waves are not as clearly known. Heppner et al. (1967) first noted the presence of such 
waves which were found to occur within a very few minutes of a bow shock crossing. These authors distinguished between frequency regions of .5 to $2.5 \mathrm{hz}$ and those greater than $3 \mathrm{hz}$ but were unable to determine the polarization of these upstream waves. Propagation from the bow shock in the whistler mode was proposed as the likely explanation for these waves, primarily because this is the only mode with high enough group velocity to propagate upstream against the solar wind which streams into the bow shock at approximately $400 \mathrm{~km} / \mathrm{sec}$. Holzer et al. (1972) studied three bow shock crossings which occurred within a time interval of eight minutes and found that in all three cases the high frequency type waves exhibited right hand polarization. This observation demonstrated that the waves were not standing with respect to the bow shock (Tidman and Northrop,1968) since a reversal of the direction of the shock motion did not produce a reversal in the observed polarization.

Russell et al. (1971) studied wave packets with frequencies near $0.4 \mathrm{hz}$ which occur upstream of and are associated with the bow shock but which are not restricted to the immediate 
vicinity of the shock. These authors also noted the occasional presence of longer wave trains with frequencies near those of the wave packets but they did not include these waves in their study. The packets were generally observed to exhibit left hand polarization, but it was argued that the polarization was right handed in the solar wind frame of reference with the observed reversal in polarization being due to movement of this reference frame past the spacecraft at the solar wind velocity. The authors rejected the explanation of whistler propagation on the basis of slightly noncircular (i.e. elliptical) polarization of the waves. Alternatively they proposed a particle origin similar to that proposed for the low frequency waves. Subsequently Wu (1972) has proposed an echo phenomenon to explain the observations of Russell et al, and Hasegawa (1972) has proposed a whistler instability.

An example illustrating the presence of both $.01-.05 \mathrm{hz}$ and $0.5-4 . \mathrm{hz}$. frequency waves is presented in Figure 1. In the remainder of this paper the terms "high" and "low" 
frequency waves will be used to refer to these two classes of waves. In the top panel 30 minutes of data is shown which was taken during an interval ending 90 minutes prinr to the subsequent observation of the bow shock at a position more than $1.5 \mathrm{R}_{E}$ downstream. The field magnitude $F$ and solar ecliptic latitude and longitude angles $\theta$ and $\emptyset$ are plotted for field averages spaced 15.36 seconds apart. Also, the square root of the sum of the squares of the three component standard deviations associated with the average are plotted as the quantity $\delta$. In the bottom panel individual measurements for the two minute interval from $19 \mathrm{~h} 44 \mathrm{~m}$ to $19 \mathrm{~h} 46 \mathrm{~m}$ are shown in the same format. The top panel clearly shows the presence of low frequency waves with periods near 50 seconds and the bottom panel illustrates the simultaneous presence of $\sim 1$ second period waves. Although both high and low frequency waves are simultaneouly present in figure 1 , either type may be seen in the absence of the other. This fact along with the existance of two characteristic frequencr ranges suggests the possibility that high and low frequency waves are two distinct phenomena with separate origins. 
The present paper reports on the further investigation of the higher frequency waves, particularly as they occur adjacent to the bow shock. It will be argued that a systematic analysis of the observations of both left and right hand polarizations confirms that they are indeed whistler mode waves.

II. Experiment

The IMP 6 (Exploxer 43) spacecraft was launched on March 13, 1971 into an eccentric orbit with the initial apogee at a geocentric distance of $33.12 \mathrm{R}_{\mathrm{E}}$ (earth radii) at a longitude $15^{\circ}$ east of the solar direction. The inclination of the orbit was $28.7^{\circ}$ and the period was 4.18 days. During the first 30 orbits bow shock crossings occurred in the northern hemisphere within $12 \mathrm{R}_{E}$ of the ecliptic plane between the solar ecliptic longitudes of $250^{\circ}$ and $35^{\circ}$. The spacecraft was spin stabilized with spin axis approximately perpendicular to the ecliptic plane. The spin period was 11.1 seconds 
during the first six months of the lifetime. The spacecraft was designed to operate in 400 and 1600 bit per second telemetry modes but since it has operated virtually exclusively in the higher mode during the first two years of its lifetime, sampling frequencies cited below will refer to the higher mode.

The IMP 6 magnetometer experiment (Seek et al, 1973) consisted of a triaxial fluxgate magnetometer with one sensor parallel and two sensors perpendicular to the spacecraft spin axis. The sensor were mounted at the end of a 13 foot boom to minimize the effects of spacecraft magnetic fields. Prelaunch testing indicated that any spacecraft field was less than $0.1_{\gamma}$ at the sensor position (Harris, 1972).

The experiment operated in any one of the four ranges $\pm 16, \pm 48, \pm 144$ and $\pm 432 \gamma$. The three orthogonal sensors were sampled within an interval of $2.0 \mathrm{milliseconds}$ and successive vector samples were taken at 80 millisecond intervals. The analog outputs from each sensor were digitized onboard the spacecraft with an 8 bit A-D converter. Full 8 bit words were telemetered to the ground every 16 measurements ( $1.28 \mathrm{sec})$ but for the intervening measurements differences were taken 
between successive samples and only these differences were supplied to the telemetry stream. These differences were restricted to 4 bit words, thus allowing a $78 \%$ increase in the sampling rate for the same telemetry assignment. Summing these differences during ground processing allowed reconstruction of the full bit words. In addition, the use of 16 differences introduced redundancy since the sum of 16 differences should be identical to the following full bit word. Rare instances when this sum did not yield the next full bit word indicated an inconsistency due either to telemetry noise or to exceeding the capacity of the 4 bit difference. Instances of exceeding the capacity of the four bit word were minimized by automatically switching the instrument to a less sensitive range when this capacity was exceeded. The instrument also automatically switched ranges according to criteria based on field magnitude. Return to a more sensitive range required both low enough fields and small enough differences (Seek et a1., 1973). Quantization step size due to digitization in the four ranges was $\pm .06, \pm .19, \pm .56 \pm .69 \gamma$. 
Zero levels of the sensors were determined by utilizing the spin of the spacecraft to reverse the orientation of the sensors perpendicular to the spin aixs. Every 46.5 hours the sensor set automatically "flipped" $90^{\circ}$ about the axis defined by a perpendicular sensor oriented along the radial direction. This action reversed the relative positions of two sensors parallel and perpendicular to the spin axis and allowed a determination of the zero level of the third sensor during the subsequent 46.5 hours. These zero level determinations were carried out independently for each range. The frequency of the zero determinations depended on the quietness of the ambient field and the expected time the sensor would be in a given range. Individual zero determinations were made at intervals which varied from every minute to every 10 minutes, but all data from half an orbit were combined and updated in the data processing program on a twice per orbit basis. The zero levels varied by less than $2_{\gamma}$ from their launch values during the first 16 months of operation. The useful accuracies of the zero levels after they are corrected by the above procedures are estimated as $\pm 0.1 Y$ in the lowest range and $\pm 0.3 \gamma$ in the highest range.

Every 11.7 hours a calibration field of $5 \gamma$ (50Y in the higher two ranges) was added along each sensor axis to check 
the sensitivity of the magnetometer. No change in the prelaunch values have been necessary during the first 1.5 years of operation.

The bandpass of the instrument ( $3 \mathrm{db}$ attenuation at $7 \mathrm{~Hz}$ ) was choosen to correspond to the $6.25 \mathrm{hz}$ Nyquist frequency of the experiment. This insures that the $0.5-4 . \mathrm{Hz}$ waves reported in this paper are being observed at their true frequency and that aliasing is not important.

III. Analysis and Results

Observations. The present study considered approximately 150 bow shock crossings which occurred on 19 of the first 30 orbits of IMP 6. These cases were typical high mach number. shocks with rare low mach number shocks (Fairfield, 1971; Formisano et al, 1971) with( $\left.M_{A} \lesssim 3\right)$ being eliminated from consideration. In most cases the bow shock is readily identified by an increase in the magnetic field strength by a factor of approximately 3 . In cases when the interplanetary field tends to be aligned with the shock normal, low frequency upstream waves are present (Fairfield, 1969; Greenstadt, 1970b) and the characteristic shock increase often is obscured by large amplitude waves or pulses (Greenstadt et al., 1970a, 1970b; Greenstadt, 1972). Such data sometimes occur continually or recurrently over an interval of several tens of minutes and 
the determination of the precise moment of "a shock crossing" becomes rather subjective. The present study has utilized data sampled at .08 sec intervals and the policy has been to consider as individual shock crossings those increases that are clearly separated by low magnitude interplanetary data for at least 30 seconds.

The set of shock crossings was divided into two groups of "clean" and "pulation" crossings according to the presence or absence of low frequency upstream waves and (usually equivalently) the presence or absence of a ragged and pulsating character of the data. Examples of the two types of crossings are shown in Figure 2. The example from April 20 shows a clean shock crossing occurring at $11 \mathrm{~h} 27 \mathrm{~m} 28 \mathrm{~s}$ where no low frequency upstream waves are present. The example from April 12 shows a pulsation crossing which would probably be located at 5h40m25s, however the additional low field region at $5 \mathrm{~h} 40 \mathrm{ml} 2 \mathrm{~s}$ and other variations at earlier times (not shown) makes the identification somewhat ambiguous. The presence of irregular low frequency upstream waves (periods approximately $20 \mathrm{sec}$ ) can also be seen in this example. 
For all of the shocks considered, the angle between the field (generally averaged over a 30 or 60 second interval) and the shock normal was calculated. The shock normal was computed from the average two dimensional bow shock determined by Fairfield (1971) in the three dimensional form given by Scudder et al. (1973). Figure 3 illustrates the histograms of the pulsation and clean crossings as a function of this angle between the field and the normal. The clean crossings are restricted almost exclusively to angles greater than $50^{\circ}$, whereas the pulsation crossings are primarily confined to angles less than this value. This result is consistent with the results of Fairfield (1969) and corresponds closely to the results of Greenstadt (1970b) when presented in terms of an average angle (Northrop and Birmingham, 1973). The lack of pulsation cases for low angles is due to the decreased. probability of the field falling within a small solid angle surrounding the normal direction, as well as the fact that a field aligned with the shock normal would not be expected to increase at the shock and produce the characteristic signature necessary to identify tre shock with only magnetic field data. 
Figure 2 also serves to illustrate the higher frequency waves which are the primary subject of this paper. In each of the examples of Figure 2 these waves are present throughout the upstream region though the frequency is clearly higher on April 20 than on April 12.

In many cases, however, the wave: amplitude decreases as the time from the shock crossing increases. This time duration, outside of which the wave amplitude is imperceptable on the plots, is typically less than a few minutes for the clean crossings, although occasionally the duration is of the order of hours or even tens of hours. For the pulsating crossings the frequent disappearance and reappearance of the waves makes it much more difficult to specify a time duration for the waves. To quantify this analysis the time duration adjacent to the shock crossing during which the high frequency waves persisted was scaled from each of the clean shock crossings. Since this time is presumably related to some damping length, cases of multiple crossings (within a few minutes) where the spacecraft remained in the vicinity of the shock were omitted from this analysis. In figure 4 this persistance time, $\Delta \mathrm{T}$, is plotted for the clear shocks versus the angle 
between the field and the shock normal. Although points do not appear at low angles due to the omission of the pulsating shocks, there is still a clear tendency for the waves to persist for a shorter duration upstream when the field normal angle is large. This persistance time cannot be converted to a distance from the shock since the relative velocity between the spacecraft and the moving shock is not known. To determine the frequencies of the waves adjacent to and upstream of the shock, power spectra were calculated for 130 shocks where the waves were present for an adequate amount of time. The spectra utilized one minute of data when available (30 seconds when necessary) and had 25 degrees of freedom. The analysis was carried out in a coordinate system where $\mathrm{Z}$ was the average field direction and $\mathrm{X}$ and $\mathrm{Y}$ were transverse to this direction. In addition to the four single component spectra (three components and the magnitude) the coherence and phase between two transverse components was calculated as a function of frequency (Bendat and Piersol, 1966). The coherence was invariably high near the peak in the spectrum and the corresponding phase was invariably near 
$90^{\circ}$ or $270^{\circ}$. These phases indicate either left or right hand polarization respectively as the sense of rotation of the wave vector relative to the average field direction.

Power spectra corresponding to the examples of Figure 2 are shown in Figure 5 for a field component transverse to the average field direction. The dashed Iine spectrum (April 12) exhibits a rapid decrease for frequencies above the peak. Such left handed spectra often reach the digitization noise level of $2 \times 10^{-4} \gamma^{2} / \mathrm{hz}$ for frequencies less than $6 \mathrm{hz}$. The solid line spectrum corresponding to the right hand waves is typical in that the peak is at higher frequencies than that for left hand waves. Usually the decrease in power for right hand waves is less abrupt and it seldom reaches the digitization noise level of the left handed cases. In most right hand cases there is a peak followed by a decreasing spectrum which suggests that the important part of the spectrum is covered by the instrumental range. This observation is supported by the work of Olson et al (1969) who found that the spectrum generally falls rapidly above approximately 5 or $10 \mathrm{hz}$. On rare occasions when the polarization is right handed the present work reveals 
only a minimal decrease at high frequencies (due partly to instrument attenuation) and in these rare cases there may be a significant spectral enhancement beyond $6.25 \mathrm{~Hz}$. The spectra of left hand polarized waves always decreases sharply and there is no suggestion that left handed waves have ever fallen outside of the instrumental range.

Of the 130 spectra calculated adjacent to the bow shock some showed a peak which was even more pronounced than those of Figure 5, but many others exhibited peaks which were broader and less well defined than Figure 5. In the great majority of cases, however, either left handed or right handed polarization could be identified as predominating. In a few cases there was evidence for left handed polarization at low frequencies and right handed polarization for higher frequencies.

Altogether 112 spectra were processed where a polarization and at least an approximate frequency could be determined for the upstream waves adjacent to the shock. Figure 6 illustrates the peak frequency of the waves plotted versus the angle between the field and the $X$ axis (the approximate direction of the solar wind). Right hand polarizations have been 
plotted as positive frequencies and left hand polarizations as negative frequencies. The lack of points with $|\mathrm{f}|<.3 \mathrm{hz}$ is due to the fact that the spectra computed in this study did not have adequate resolution in this low frequency regime. Furthermore, frequencies below $0.3 \mathrm{hz}$ begin to overlap the $.01-.05 \mathrm{hz}$ frequency range where most of the observed waves apparently have a different origin. Clearly an angle of $55^{\circ} \pm 5^{\circ}$ in figure 6 separates the cases of right and left polarization. Each group of polarization in figure 6 contains many cases of interplanetary to magnetosheath traversals as well as magnetosheath to interplanetary traversals. Since the interplanetary field orientation determines the sense of polarization so well and since the field orientation cannot be well correlated with the direction of shock motion, this figure can be cited as confirmation of the result of Holzer et al. (1972) that the polarization does not depend on the relative direction of motion between the shock and the satellite.

Another characteristic of prime importance in the study of waves is their propagation vector $\vec{k}$. This direction can be determined by a technique originally developed by Sonnerup and Cahill (1967) for studying the magnetopause, but 
subsequently applied to waves by many authors (e.g. Siscoe et al., 1967; Russell et a1., 1971; Holzer ët al., 1972). The procedure is to diagonalize the variance matrix in order to determine the direction of minimum variance which is assumed to be the $\pm \hat{k}$ direction of the waves. The procedure gives no information about the magnitude of $k$ and also leaves an ambiguity in the sign of $k$ which is surpressed by assuming the propagation is in the upstream direction. This variance analysis was carried out on the upstream wave events for which spectra were available. In each case the technique was applied to successive intervals of 4 seconds (several cycles of the wave) covering an interval of typically half a minute. This procedure allowed intercomparison of the computed $\hat{k}$ vectors from the various segments. In 84 cases the individually determined $\hat{k}$ vectors were judged to agree well enough among themselves (i.e.to fall roughly within a cone of $15^{\circ}$ half angle) to warrant using the event. In these acceptable cases both the angles between the field and the $\hat{k}$ vector and the $\hat{k}$ vector and the $X$ axis were averaged.

Figure 7 is a histogram showing the number of cases with different values of the angle between the upstream field vector 
and $k$. There is a strong preference for waves to propagate at an angle between $20^{\circ}$ and $40^{\circ}$ to the field with a clear tendency for the waves to neither propagate along nor perpendicular to the field. The fact that the waves generally propagate preferentially at an angle within $40^{\circ}$ of the field direction suggests that the $\hat{\mathbf{k}}$ vector might possibly be substituted for $F$ in ordering the data of Figure 6 .

Figure 8 is the figure similar to Figure 6 only with the abscissa now being the angle between $\hat{k}$ and the approximate solar wind direction, $x$. The points represent a subset of those of Figure 6 corresponding to those cases where a $\hat{k}$ vector couid be determined. The crosses represent occurrences of the waves at locations more than 2 hours upstream of the nearest shock crossing. Indeed the $k-x$ angle orders the data about as well as the F-x angle of Figure 6, particularly considering the greater uncertainties in the determination of $\hat{k}$. Now a $\mathrm{kx}$ angle of about $40^{\circ}$ separates the regions of positive frequency ( $r$ ight hand polarization) and negative frequency (left hand polarization). It also appears that right hand waves occur at frequencies above $2.5 \mathrm{hz}$, whereas 
left.hand waves do not (do not fall below $-2.5 \mathrm{hz}$ in Figure 8). It will be argued in the next section that Figure 8 is the key to confirming the origin of the waves as whistler mode propagation from the bow shock.

It should be pointed out that due to the geometry of the bow shock there is a tendency for the shock normals to point in a generally upstream direction. This tendency means that there is a strong statistical tendency toward the condition for low frequency upstream waves and pulsating shocks (the field aligned generally with $\hat{n}$ : see Figure 3 ) to occur at the same time as the condition for left handed high frequency waves (the field generally aligned with $\mathrm{x}$ : see Figure 6). This statistical correlation can be violated, however, and pulsation shocks can accompany right handed waves and clean shocks can accompany left handed waves. This latter case is illustrated by Figure 9 which shows the detailed data for a shock crossing on April 3, 1971. These data appear to be typical of clean shock crossings but they are unusual in that the upstream waves are left hand polarized rather than right hand polarized as they are for most clean shock crossings. 
By looking at the various angles we can understand this polarization (see insert in Figure 9). The field makes an angle of $50^{\circ}$ with the shock normal which is apparently just large enough to exclude the low frequency waves and create the "clean" shock. At the same time the field (which happens to be oriented almost perpendicular to the average spiral angle) makes an angle of only $37^{\circ}$ with the $\mathrm{X}$ axis ( $\mathrm{kx}$ angle is $12^{\circ}$ ) and therefore the waves are left handed. It appears that exceptions to the general correlation of pulsating crossings with left handed high frequency waves and clean crossings with right handed high frequency waves can be explained in this manner. There appears to be no reason to suggest that the statistical correlation has any independent significance.

To illustrate that high frequency waves are related to shocks other than the earth's bow shock, Figure 10 presents the data at the time of two interplanetary shocks. On May 30 a very clear wave train is apparent ahead of the shock and power spectra indicate a clear peak at $1.9 \mathrm{~Hz}$ with right 
handed polarization. On May 17 , higher frequency $(4.2 \mathrm{~Hz})$ right handed waves are present upstream from the shock. In these two cases $\hat{k}$ makes angles of $46^{\circ}$ and $65^{\circ}$ respectively with the solar wind direction. At the time of three of four other available interplanetary shock waves, similar waves are seen, although in two cases the amplitude is too small and the duration ( $\sim 4$ seconds) too short to permit further analysis. The remaining analyzable case had a frequency of $1.0 \mathrm{~Hz}$, exhibited right hand polarization, and had a kx angle of $73^{\circ}$. Only on one interplanetary shock with the field aligned near the shock normal were no waves observed. Interpretation. The association of $0.5-4$. hz waves with the bow shock and their apparent damping with distance from it suggests that the waves may be propagating away from the shock. As was pointed out by Heppner et al. (1967), the whistler mode is an obvious candidate for their explanation since it has a group velocity greater than the solar wind velocity. The wave frequencies under consideration are well below the electron and ion plasma frequencies and the electron gyrofrequency but above the ion gyrofrequency so the simple cold plasma dispersion relation(e.g. Stix 1962) for right hand polarized whistlers becomes 


$$
\frac{c^{2} k^{2}}{\omega^{\prime}}=\frac{{ }^{\omega} \mathrm{pe}^{2}}{\left(\omega^{\prime}-\Omega_{\mathrm{e}} \cos \theta\right)}
$$

where $\omega^{\prime}$ is the wave frequency in the plasma frame, $\omega_{p e}$ is the electron plasma frequency $\left(\omega_{p e}^{2}=\frac{4 n_{e}}{m_{e}}\right), \Omega_{e}$ is the electron gyrofrequency $\left(\Omega_{e}=\frac{e F}{m_{e} c}\right), \theta$ is the propagation angle with respect to the field direction (the angle in Figure 7 ), $\mathrm{n}$ is the plasma density and $\mathrm{k}=2 \pi / \lambda$.

Waves observed at the spacecraft will have undergone a doppler shift due to motion of their frame of reference past the spacecraft. They will be detected at frequency $w$ where

$$
\omega=\omega^{\prime}+\overrightarrow{\mathbf{k}} \cdot \overrightarrow{\mathrm{v}}_{\mathrm{SW}}
$$

and $\mathrm{V}_{\mathrm{SW}}$ is the solar wind velocity. With the assumption that propagation is in the upstream direction, we may rewrite 2 as

$$
f=f^{\prime}-\frac{V_{S W}}{\lambda} \cos (k x)
$$

where the $\mathrm{kx}$ angle is that of Figure 8 . The observed frequency $f$ will be the plasma frame f' reduced by 
a factor depending on the solar wind velocity, the wavelength and the direction of propagation. Physically a negative $f$ in equation $2^{\prime}$ means that the wave whose perturbation vector traces a right handed helix as it propagates upstream in the plasma frame is being convected downstream past the spacecraft in such a way that the observed polarization is reversed. For this reversal to take place the phase velocity of the wave must be less than the solar wind velocity but at the same time the group velocity must be greater than the solar wind velocity to enable the waves to propagate away from the shock. (Actually the above is strictly true only at the subsolar point for propagation in an exactly upstream direction. The problem should actually be approached three dimensionally in which case the shock normal is introduced into the problem and the component of the group velocity along the normal is required to exceed the solar wind velocity component along the normal. Only the one dimensional argument will be presented here, but it should suffice for the order of magnitude arguments given below.) 
The solid curve in Figure 11 is a plot of the dispersion relation of equation 1 ( $f$ in hertz vs $k$ ) for a typical set of solar wind parameters. In addition the phase velocity $\left(V_{p h} \equiv \frac{\omega}{k}\right)$ and group velocities $\left(V_{g} \equiv \frac{d \omega}{d k}\right)$ have been calculated from the dispersion relation and are shown as dashed 1 ines. These velocities correspond to the scale on the right. It is of interest to examine the phase and group velocities relative to a typical solar wind velocity of $400 \mathrm{~km} / \mathrm{sec}$ to see whether the conditions of $\mathrm{V}_{\mathrm{g}}>400 \mathrm{~km} / \mathrm{sec}, \mathrm{V}_{\mathrm{ph}}<400 \mathrm{~km} / \mathrm{sec}$ can be met simultaneously. For $\mathrm{k}>.65\left(\lambda<10 \mathrm{~km} ; \mathrm{f}^{\prime}>40 \mathrm{hz}\right)$ the group velocity is seen to be less than $400 \mathrm{~km} / \mathrm{sec}$ and waves could not even propagate away from the shock. For .09<k<.65 $(10<\lambda<70 \mathrm{~km}$;

$\left.5.7<\mathrm{f}^{\prime}<40 \mathrm{hz}\right)$ the waves could propagate upstream, but their phase velocity is also larger than the solar wind velocity and consequently no left hand polarizations would be observed. For $\mathbf{k}<.043\left(\lambda>148 \mathrm{~km} ; \mathrm{f}^{\prime}<1.4 \mathrm{hz}\right)$ the group velocity is again too low to allow upstream propagation. (Care must be exercized at very low frequencies where the neglected ion term in the dispersion relation becomes important). 
On1y in the very 1 imited frequency range where $.043<k<.09$ $\left(70<\lambda<148 \mathrm{~km} ; 1.4<\mathrm{f}^{\prime}<5.7 \mathrm{hz}\right)$ would we expect to see both left and right handed polarization because only in this frequency range is the group velocity greater than $400 \mathrm{~km} / \mathrm{sec}^{\circ}$ and the phase velocity less than $400 \mathrm{~km} / \mathrm{sec}$. This theoretical frequency range corresponds very closely to the frequency range where both left and right handed waves are observed.

Identification of this range of frequencies where left handed waves can be observed is equivalent to saying $\frac{\mathrm{V} S W}{\lambda}>\mathrm{f}^{\prime}\left(\right.$ see $\left.2^{\prime}\right)$. The cosine in $2^{\prime}$ will actually determine which of the terms on the right hand side of $2^{\prime}$ is larger. and which polarization will be observed at a particular time. This prediction is exactly the result illustrated in Figure 8 which shows that right handed waves are observed for large angles (small values of the cosine) and left handed waves for small angles (large cosines).

On the basis of Figure 8 it can be said that zero frequency waves should occur at a $\mathrm{kx}$ angle of $\sim 40^{\circ}$ when the phase velocity of the wave equals the component of the solar 
wind velocity in the $\hat{k}$ direction. Assuming a solar wind velocity of $400 \mathrm{~km} / \mathrm{sec}$, we obtain an average phase velocity of

$$
\frac{\omega}{\mathrm{k}}=400 \cos 40^{\circ}=306 \mathrm{~km} / \mathrm{sec} .
$$

Referring to Figure 11, we find that this velocity corresponds to a frequency in the plasma frame of $3.2 \mathrm{hz}$ which in turn, through the dispersion relation, corresponds to a wavelength of $95 \mathrm{~km}$.

It is interesting to note that this frequency of $3.2 \mathrm{hz}$ is the lower hybrid frequency in an average field of $5 y$, raising the question of whether the lower hybrid frequency is a significant frequency. In principle, it is possible to see whether the waves always occur at the lower hybrid frequency by solving equations 1 and 2 simultaneously with the measured parameters from individual cases. In practice, this solution involves a cubic equation whose solution has not yet been attempted. Individual cases have been numerically investigated, however, (using the measured solar wind parameter obtained from by the Los Almos plasma experiment on 
board the same spacecraft) and it appears that although individual determinations of the plasma frame frequencies would cluster about the lower hybrid frequency, they do not correspond to it on a case by case basis.

The explanation of whistler propagation is apparently successful in explaining the observed dependence of polarization on $\mathrm{kx}$ angle, but it remains to see whether other observed characteristics are consistent with this interpretation. Clearly, the solar wind velocity, density, field magnitude and propagation direction should determine the observed frequencies if the waves are whistlers, so it is of interest to determine how sensitive the frequency is to variations in these parameters.

Figure 12 is a figure similar to Figure 11 , only it is plotted for the more limited frequency range of interest and for two sets of observed parameters. The solid curves correspond to density values of $3 / \mathrm{cc}$ and the dashed curves to densities of $12 / \mathrm{cc}$. The lower two sets of curves represent the dispersion relations with the upper curve in each set corresponding to a field propagation angle of $20^{\circ}$ and the 
lower curve to an angle of $40^{\circ}$. The upper four sets of curves correspond to phase and group velocities calculated from the dispersion curves at the bottom with the two curves in each set corresponding to the two propagation directions. The vertical lines denote the regions where $\mathrm{V}_{\mathrm{ph}}<400 \mathrm{~km}<\mathrm{v}_{\mathrm{g}}$ for each set of parameters. If the waves are to continually satisfy this condition (and there is no "a priori" reason they should do so) they must assume lower frequencies (and longer wavelength) for low densities and higher frequencies (and shorter wavelengths for high densities. The curves of Figure 12 are all drawn for a field strength of $5 \gamma$ but it is easily shown that for a given $\mathrm{k}$ the plasma frame frequency is proportional to the field strength. This means that such curves calculated for individual cases while always approaching zero at low frequencies will increase or decrease in proportion to the field strength. This fact plus the relative sensitivity of the curves to density and propagation direction make it quite plausible that the scatter of points in Figure 8 is caused by variations in these parameters. 
Another observed characteristic of the upstream wave is the fact that left handed waves were not found to exceed $2 \mathrm{hz}$, whereas right handed waves were seen at least as high as $4.5 \mathrm{hz}$ and perhaps extending to higher frequencies. This observation can be explained in terms of the whistler interpretation when it is recalled that the left hand frequency, is produced by the $V / \lambda$ term in equation $2^{\prime}$. Since for typical solar wind conditions we found that $\lambda$ was near $100 \mathrm{~km}$, this term is typically of the order of 4hz. It in turn is decreased by adding the first term of $2^{\prime}$ which is of the opposite sign, and typically has a magnitude between 2 and $3 \mathrm{hz}$. Thus the sum is typically between one and two hertz and only for unusually high solar wind velocities or unusually short wavelength waves can the left handed waves appear at higher frequencies. In fact all of the left handed cases in figure 8 correspond to rather average solar wind densities and field strengths and therefore would not be expected to have higher frequencies. The highest velocities associated with left handed waves were 500 and $660 \mathrm{~km} / \mathrm{sec}$ and these correspond to 2 of the 3 highest frequencies (most 
negative $f^{\prime} s$ ) observed near the shock in Figure 8 .

It is also of interest to look more closely at the one point in Figure 8 that is in apparent disagreement at $f=1.1, \quad \mathrm{kx}=16^{\circ}$. This point corresponds to an unusually low density of 3 , a field strength of $4.3_{y}$ and a fieldpropagation angle of $49^{\circ}$. The simultanious solution of equations 1 and 2 reveals that the observed frequency of $1.1 \mathrm{hz}$ is the sum of an unusually large plasma frame frequency of $10.0 \mathrm{hz}$ and a doppler shift term of $-8.9 \mathrm{hz}$ whose large magnitude is due to an unusually short wavelength of $50 \mathrm{~km}$. The corresponding phase velocity is an unusually high $520 \mathrm{~km} / \mathrm{sec}$ which is larger than the simultaneously observed solar wind velocity of $470 \mathrm{~km}$, thus explaining the absence of a polarization reversal and why the right handed polarization was observed.

It should be pointed out that the whistler propagation explanation for the observed waves is not dependent on the shock other than through the assumption that the waves are propagating away from the shock. It is also true that little has been said about why bands of frequencies are observed 
other than to note that the plasma frame frequencies are near the lower hybrid frequency but not necessarily at it. One possible explanation of preferred frequencies is that they are generated with wavelengths which correspond to the scale of the shock thickness (e.g., Tidman and Kral1, 1971).

Ful1 consideration of this question will be deferred for future work, but here we note that theoretical estimates of the thickness of the shock $\frac{2 \pi c}{\omega_{e}}$ or $\frac{2 \pi c}{\omega_{i}}$ are of the order of $15 \mathrm{~km}$ and $700 \mathrm{~km}$ and thus span the range of wavelengths for the observed whistler waves.

To further check the plausibility of this idea the simultaneous solution to equations 1 and 2 have been obtained for three interplanetary shocks where unusually narrow band upstream waves can be studied. Two of these shocks have been studied by Chao et al. (1973) and their orientation and velocity in the solar wind frame are known. Once the velocity of the shock relative to the spacecraft is known, a thickness can be calculated by noting the traversal time as deduced from the magnetic field data. This shock thickness can be compared with the independent calculation of the 
wavelength. The relevant parameters are listed in table 1

where $\Delta t$ is the duration of the crossing and $L$ the shock thickness.

Table 1. Interplanetary Shocks

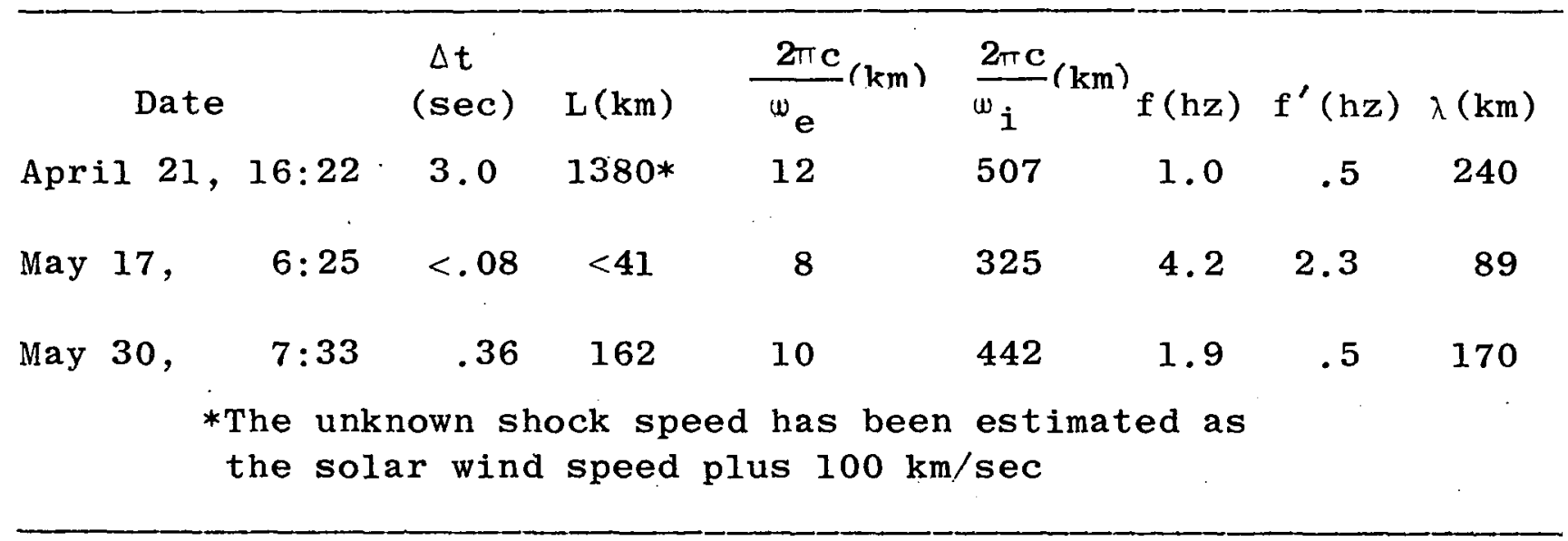

There is a general correspondence between $\lambda$ and the shock thickness with the smallest $\lambda$ corresponding to the smallest $L$ and the largest $\lambda$ corresponding to the largest $L$.

As another check on the whistler theory, we note that waves propagating away from interplanetary shocks will have a component of their $k$ vector in the solar wind direction except perhaps in cases where the outward shock normal makes a large angle relative to the radial from the sun. Having $k$ and $V$ in the same general direction is the opposite situation from that of the 
bow shock and requires that the sign of the last term in equation $2^{\prime}$ be reversed. This implies that only right hand polarization would be expected to be observed ahead of interplanetary shocks with conventional geonetry. All three measurements of the polarizations ahead of interplanetary shocks . have in fact been right handed.

Another question concerning upstream whistler waves is that of their damping. Figure 4 demonstrates that damping often takes place within a few minutes of the shock or, alternatively, in something like 100 cycles of the wave. There appears to be a tendency for larger field-normal angles to correspond to higher frequencies so one possible explanation of the slope in figure 4 is that the damping generally takes place over a given number of cycles but for larger Fn angles the cycles are shorter and the time interval during which the waves persist is smaller. This cannot be the complete explanation, however, because instances of high frequency waves occurring near spacecraft apogee in the sunward direction are found on occasion. In these instances, if we assume the waves are the same as those found near the bow shock, they 
must have propagated many earth radii and undergone minimal damping over many hundreds of cycles.

Cyclotron and Landau damping are candidates for damping upstream whistler waves since the propagation is not field aligned and the electric field will have a component both parallel and perpendicular to the background field. In these resonant interactions between waves and electrons, energy can be exchanged between the waves and the particles. Extraction of wave energy from the particles results in damping but it should be noted that the energy of electrons moving away from the bow shock can also be converted into waves under the appropriate circumstances. In this sense it is not completely clear that whistler mode waves are propagating from the bow shock rather than being generated by electrons moving upstream from the shock.

The question of non-linear Landau damping of electrostatic waves has been studied by $0^{\prime}$ Neil (1965) and the non-linear damping of whistler waves has been studied by Palmadesso and Schmidt (1971) and Palmadesso (1972). These results offer a promising explanation of the lack of damping. These authors have shown that after an initial partial damping of a large amplitude waves, the electron distribution becomes modified in a 
manner such that it is unable to easily extract additional

energy from the waves. For a monochromatic wave the damping ceases completely and for a more realistic wave spectrum the damping times are significantly lengthened over that expected on the basis of linear theory. If these theories prove applicable it is perhaps more appropriate to ask the question of why the waves are usually damped quickly rather than persisting for long distances.

Discussion. In reexamining the previously reported results in the light of the current observations it is clear that the waves discussed in the present paper are of the same type as those first noted by Heppner et al. (1967). These authors distinguished between one class of waves which occurred in packets and exhibited a rather sharp cutoff in frequency between .5 and $2.5 \mathrm{hz}$ and a second class of more continuous waves at higher frequencies. The waves with frequencies less than $2.5 \mathrm{hz}$ can be identified with the left handed waves of the present study and the higher frequency waves with the right handed waves. Gradations between the two categories which were not evident in the earlier study are now apparent so there no longer appears to be good reason to distinguish between these two categories, particularly since the whistler explanation seems applicable to all waves. It is true, however, that left handed waves 
often, but not always, occur in packets. This fact may be related to the fact that such wave packets invariably occur in the presence of low frequency waves which are continuously changing the orientation of the "ambient" field. It also may be due to the fact that whistler waves are acting in the shock formation process in the manner described by Hasegawa (1972). The packets away from the shock studied by Russell et al. (1971) may be a different phenomena than the waves studied in the present paper. The theory of $W u$ (1972) is more applicable to these packets, whereas the theory of Hasegawa (1972) appears to be more applicable to the waves of the present study. The three cases of Holzer et al. (1972) exhibited right hand polarization and occured with the upstream field nearly perpendicular to the solar wind direction and hence they fit naturally into the results of the present paper. IV. Summary and Conclusions Waves of frequencies $0.5-4$. hz persist upstream of the earth's bow shock for intervals which are usually less than a few minutes in time but which occasionally are much longer. The waves propagate obliquely to the field at an 
angle which is typically between $20^{\circ}$ and $40^{\circ}$. The waves may exhibit either. left handed or right handed polarization : with the particular polarization being controlled by the propagation direction (alternatively the field direction). relative to the solar wind direction. The power spectra of left handed waves decreases sharply beyond about $2 \mathrm{hz}$ and predominant frequencies of left hand waves have never been seen beyond $2.5 \mathrm{hz}$. Spectra of right handed waves often have peaks below $4 \mathrm{hz}$, but the spectrum decreases less rapidly than left handed waves. Similar waves appear to be present ahead of interplanetary shock waves.

The cold plasma dispersion relation for right handed electron whistler waves is shown to be consistent with the observations in all respects. This equation predicts that waves of the observed frequencies could be detected upstream if they were emitted from the bow shock. It further predicts that such waves should be either left or right hand polarized depending on the propagation direction relative to the solar wind in just the manner observed. The left handed waves are due to doppler shifting between the plasma frame of 
reference and the observation frame. The $2.5 \mathrm{hz}$ upper limit for left hand waves is due to the fact that wavelengths are invariably near $100 \mathrm{~km}$ for normal solar wind conditions, whereas unusually small wavelengths or large solar wind velocities would be needed to produce greater doppler shifts and higher left handed frequencies. It is tentatively suggested that the observed spectral peaks correspond to waves whose wavelength of approximately $100 \mathrm{~km}$ is related to the shock thickness. 
Acknowledgements

The author gratefully acknowledges the support of Dr. N.F. Ness who is principal investigator on the IMP-6 magnetic field experiment and Mr. J.B. Seek and Mr. John Scheifele who carried out the engineering aspects of the experiment. Grateful thanks are also due Dr. S.J. Bame and colleagues at the Los Almos Scientific Laboratory who supplied the plasma data which was invaluable in carrying out this work.

The author also appreciates many stimulating discussions and helpful suggestions from numerous colleagues at Goddard Space Flight Center and the University of Maryland. Although the entire list of helpful people is too extensive to enumerate, T.J. Birmingham, and T.G. Northrop of Goddard and P.J. Palmadesso of Maryland should be cited for their particular interest. 


\section{References}

Barnes, Aaron, "Theory of generation of bow-shock-associated hydromagnetic waves in the upstream interplanetary medium", Cosmic Electrodynamics, 1, 90-114, 1970.

Bendat, Julius $S$. and Allan G. Piersol, "Measurement and analysis of random data", John Wiley \& Sons, Inc. New York, 1966.

Fairfield, D.H., "Bow shock associated waves observed in the far upstream interplanetary medium", J. Geophys. Res., 74, 3541-3553, 1969 .

Fairfield, Donald H., "Average and unusual locations of the earths magnetopause and bow shock", J. Geophys Res., 76, 6700-6716, 1971 .

Formisano, V., P.C. Hedgecock, S. Moreno, J. Sear, D. Bollea, "Observations of earth's bow shock for low mach numbers," Planet. Space Sci, 19, 1519-1531, 1971.

Greenstadt, E.W., I.M. Green, G.T. Inouye, D.S. Colburn, J.H. Binsock, and E.F. Lyon, "Dual satellite observations of earth's bow shock I: The thick pulsation shock," cosmic Electrodynamics, 1, 160-177, 1970a.

Greenstadt, E.W., I.M. Green, G.T. Inouye, D.S. Colburn, J.H. Binsach, and E.F. Lyon, "Dual satellite observations of earth's bow shock II: Field aligned upstream waves," Cosmic Electrodynamics, $1,279-296,1970 \mathrm{~b}$.

Greenstadt, E.W., I.M. Green, G.T. Inouye, D.S. Colburn, J.H. Binsach, and E.F. Lyon, " Dual satellite observations of earth's bow shock III: Field determined shock structure, "n Cosmic Electrodynamics,"1, 316-327, 1970c.

Greenstadt, E.W., "Observation of nonuniform structure of the earth's bow shock correlated with interplanetary field orientation", J. Geophys. Res., 77, 1729-1738, 1972.

Harris, C.A., "IMP-I spacecraft final magnetic tests," Goddard Space Flight Center publication X-325-72-183, 1972.

Hasegawa, A. "Excitation and propagation of an upstreaming electromagnetic wave in the solar wind," J. Geophys. Res., 77, 84-90, 1972 . 
Heppner, J.P., M. Sugiura, T.L., Skillman, B.G. Ledley, and M. Campbe11, "OGO-A magnetic field observations"; J. Geophys. Res., 72, 5417-5471, 1967 .

Holzer, Robert E., Theodore G. Northrop, John V. Olson and Christopher T. Russe11," J. Geophys. Res., 77, 2264-2273, 1972 .

Northrop, T.G., and T.J. Birmingham, "On Greenstadt's binary index criterim," J. Geophys. Res., 78, 2308-2310, 1973.

Olson, John V., Robert E. Holzer and Edward.J. Smith,"Highfrequency magnetic fluctuations associated with the earth's bow shock", J. Geophys. Res., 74, 4601-4617, 1969.

O'Neil, T., "Collisionless damping of non-linear plasma oscillations", Phys. Fluids, $\underline{8}, 2255-2262,1965$.

Palmadesso, P.J., "Resonance, particle trapping, and Landau damping in finite amplitude obliquely propagating waves," Physics of Fluids, 15, 2006-2013, 1972.

Palmadesso, P., and G. Schmidt, "Collisionless damping of a large amplitude whistler wave," Physics of Fluids, 14, 1411-1418, 1971 .

Russe11, C.T., D.D. Childers and P.J. Coleman, Jr.,"OGO-5 observations of upstream waves in the interplanetary medium; discrete wave packets," J. Geophys. Res., $\underline{76}$, $845-861,1971$.

Scudder, J.D., D.L. Lind and.K.W. Ogilvie, "Electron Observations in the solar wind and magnetosheath," J. Geophys. Res. in press 1973 .

Seek, J.B., J.L. Scheifele, and N.F. Ness, "GSFC magnetic field experiment Explorer 43," to be published 1973.

Siscoe, G.L., L. Davis Jr., P.J. Coleman, Jr, E.J. Smith, and D.E. Jones, "Shock aligned magnetic oscillations in the magnetosheath: Mariner 4, J. Geophys. Res., 72, 5524_5530, 1967 .

Sonnerup, B.U.O., and L.J. Cahill Jr., "Magnetopause structure and attitude from Explorer 12 observations," J. Geophys. Res., 72, 171-183, 1967 . 
Stix, T.H., "The theory of plasma waves," McGraw-Hill Book Company, New York, 1962.

Tidman, D.A., and N.A. Krall, Shock waves in collisonless plasmas, John Wiley and Sons, New York, 1971.

Tidman, D.A., and T.G. Northrop, "Emission of plasma waves by the earth's bow shock", J. Geophys. Res., 73, 1543-1553, 1968 .

Wu, C.S., "Theory of discrete wave packets in the solar wind," J. Geophys. Res., 77, 575-587, 1972. 


\section{FIGURE CAPTIONS}

Figure 1 - Illustrating the two classes of waves often seen upstream of the earth's bow shock.

Figure 2 - Illustrating a "clean" shock crossing without low frequency waves (top) and a "pulsation" crossing in the presence of low frequency waves.

Figure 3 - The number of "clean" and "pulsation" shock crossings as a function of the angle between the field and the shock normal. "Clean" crossings are confined largely to angles greater than $50^{\circ}$ and pulsation crossings and upstream waves to angles less than $50^{\circ}$.

Figure 4 - Persistencetime of high frequency waves adjacent to the bow shock as a function of the angle between the field and the shock normal.

Figure 5 - Power spectra of a field component transverse to the average field direction for the examples of Figure 2. The April 12 example exhibits left handed polarization whereas the April 20 case exhibits right handed polarization.

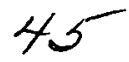


Figure 6 - Observed frequency of upstream waves as a function of the angle between the field and the approximate solar wind direction. Positive frequencies correspond to right hand polarizations and negative frequencies to left hand polarization.

Figure 7 - Number of cases of upstream waves propagating at various angles to the upstream field.

Figure 8 - Observed frequency of upstream waves as a function of the propagation direction relative to the approximate solar wind direction. Positive frequencies correspond to right hand polarization and negative frequencies to left hand polarization.

Figure 9 - Clean shock crossing on April 3, 1971。 The data appear to be typical but in fact are unusual since the upstream waves are left hand polarized rather than right hand polarized as in most clean shock crossings.

Figure 10- Illustrating the presence of waves ahead of two interplanetary shocks. Calculated shock thickness and wavelengths are $162 \mathrm{~km}$ and $170 \mathrm{~km}$ on May 30 and $<41 \mathrm{~km}$ and $89 \mathrm{~km}$ on May 17 . 
Figure 11 - The cold plasma dispersion relation (solid curve) and associated phase and group velocities (dashed curves) for typical solar wind conditions. The shaded region indicates where left and right handed whistler waves could be observed upstream from the earthis bow shock.

Figure 12 - Cold plasma dispersion relations (bottom) and phase and group velocities (top) for two values of upstream densities. The shaded areas represent the regions bounded by field-propagation angles of $20^{\circ}$ (top 1 ine of each pair) and $40^{\circ}$. Vertical lines help designate the regions where group values are greater than a typical solar wind velocity and phase velocities are less than the solar wind velocity. 


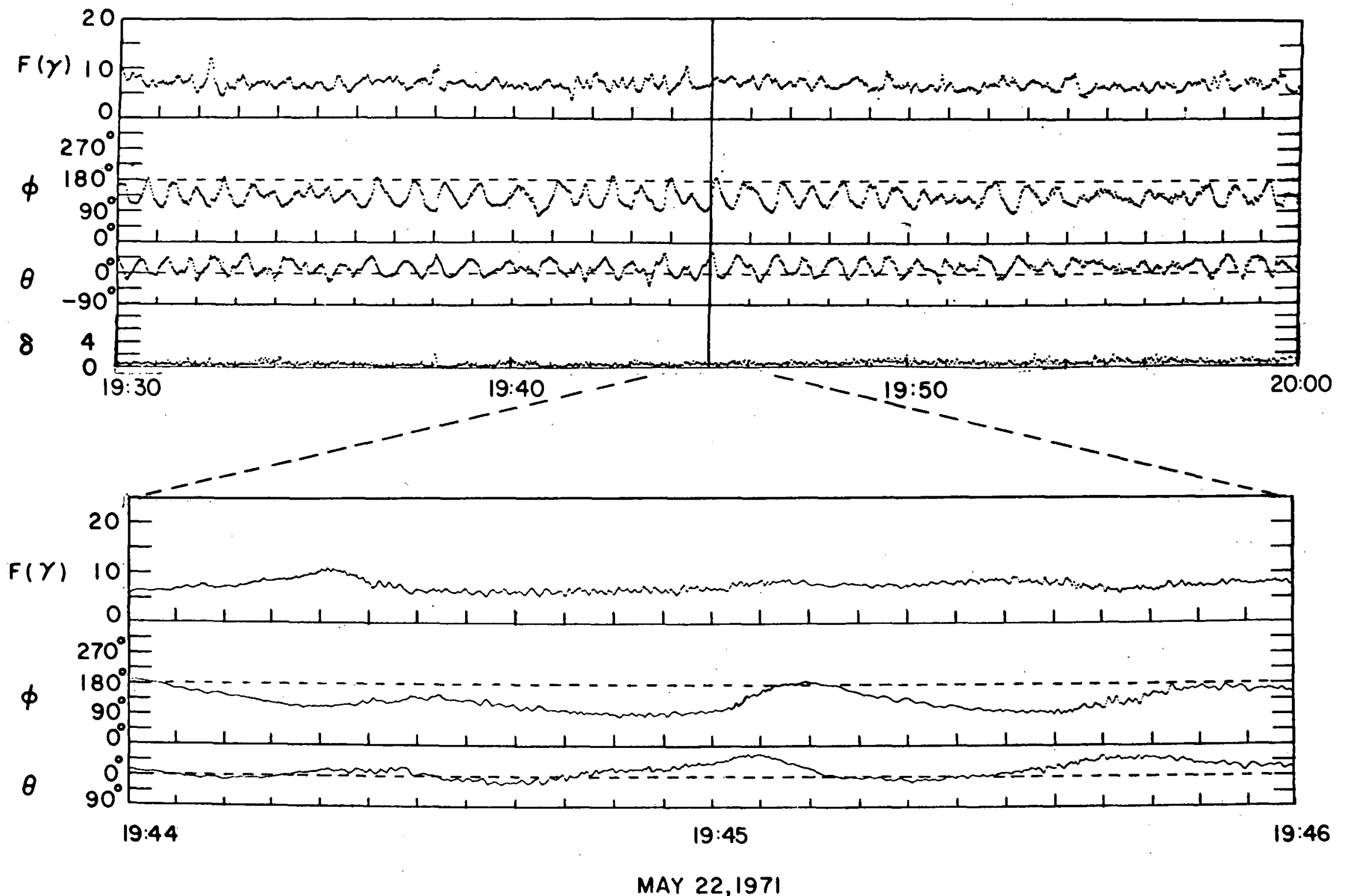

Figure 1 

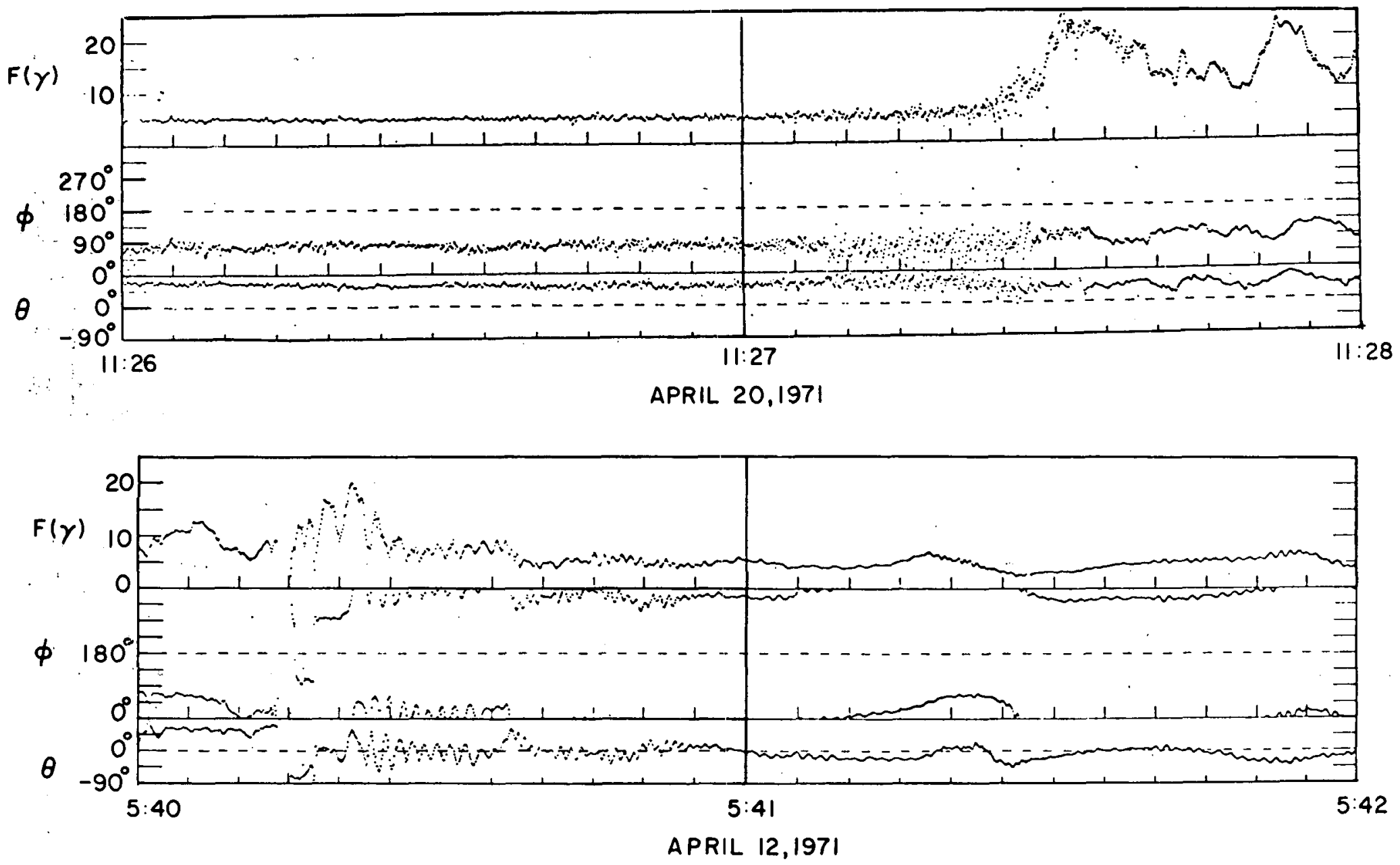

Figure 2 


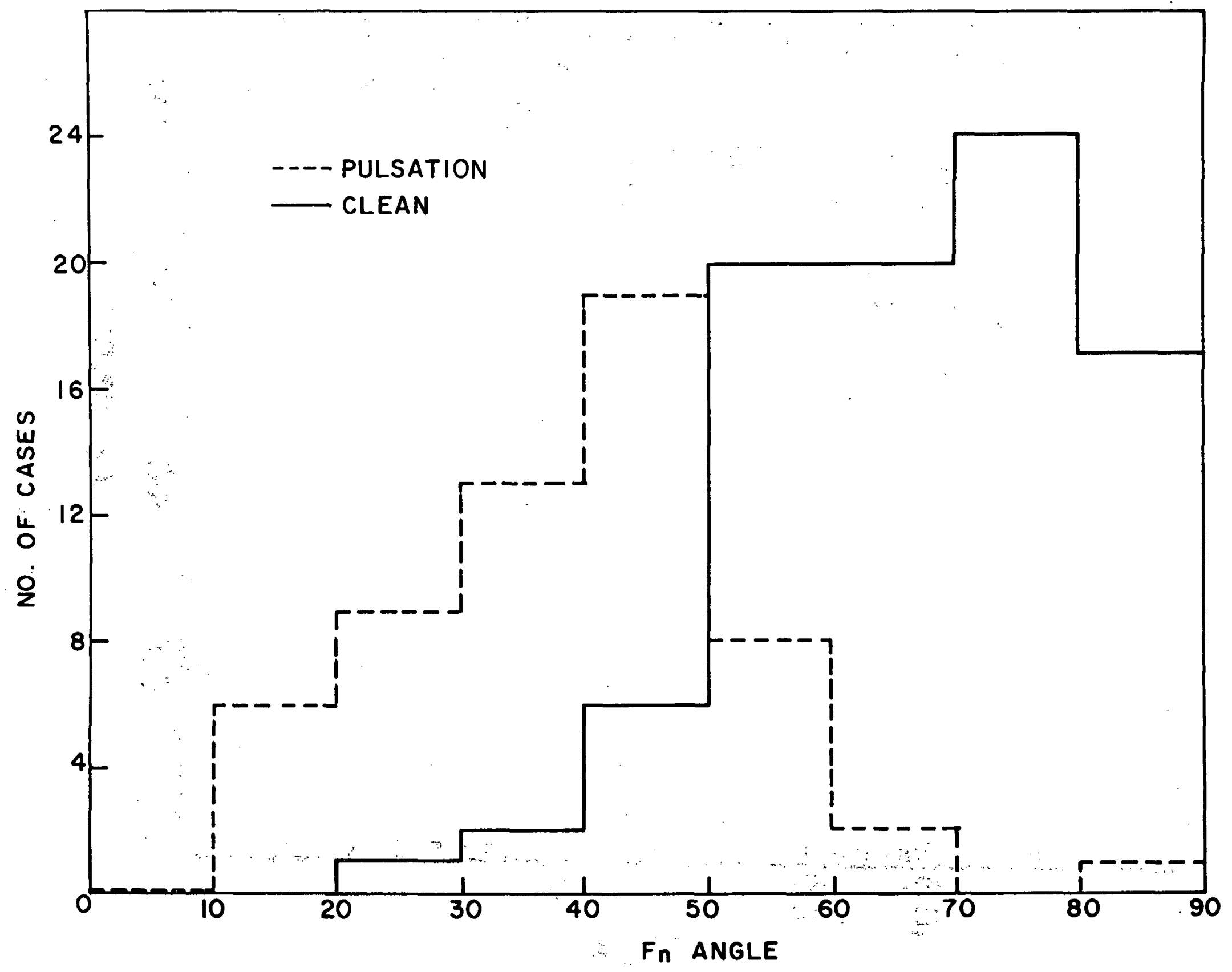

Figure 3 


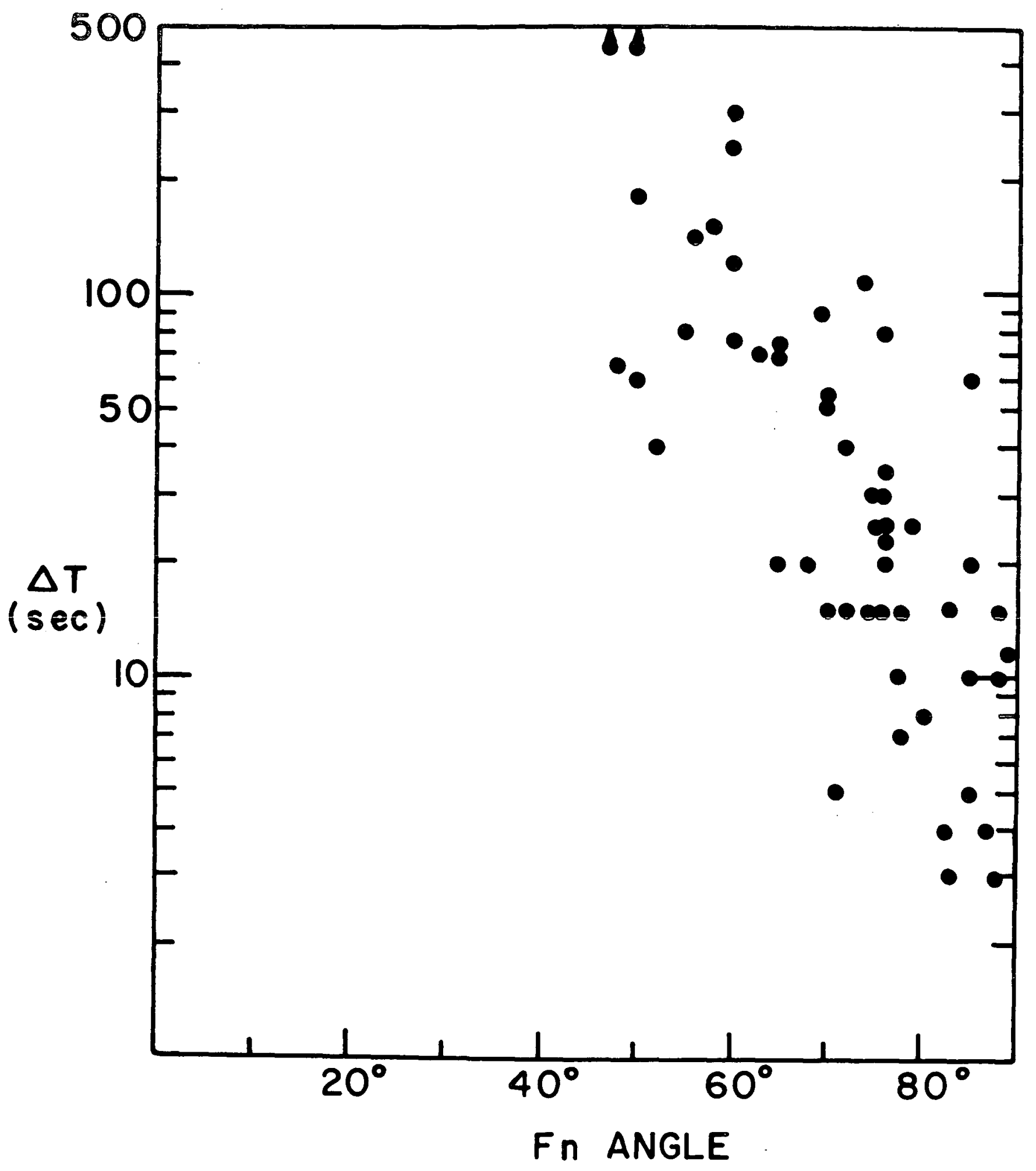

Figure 4 


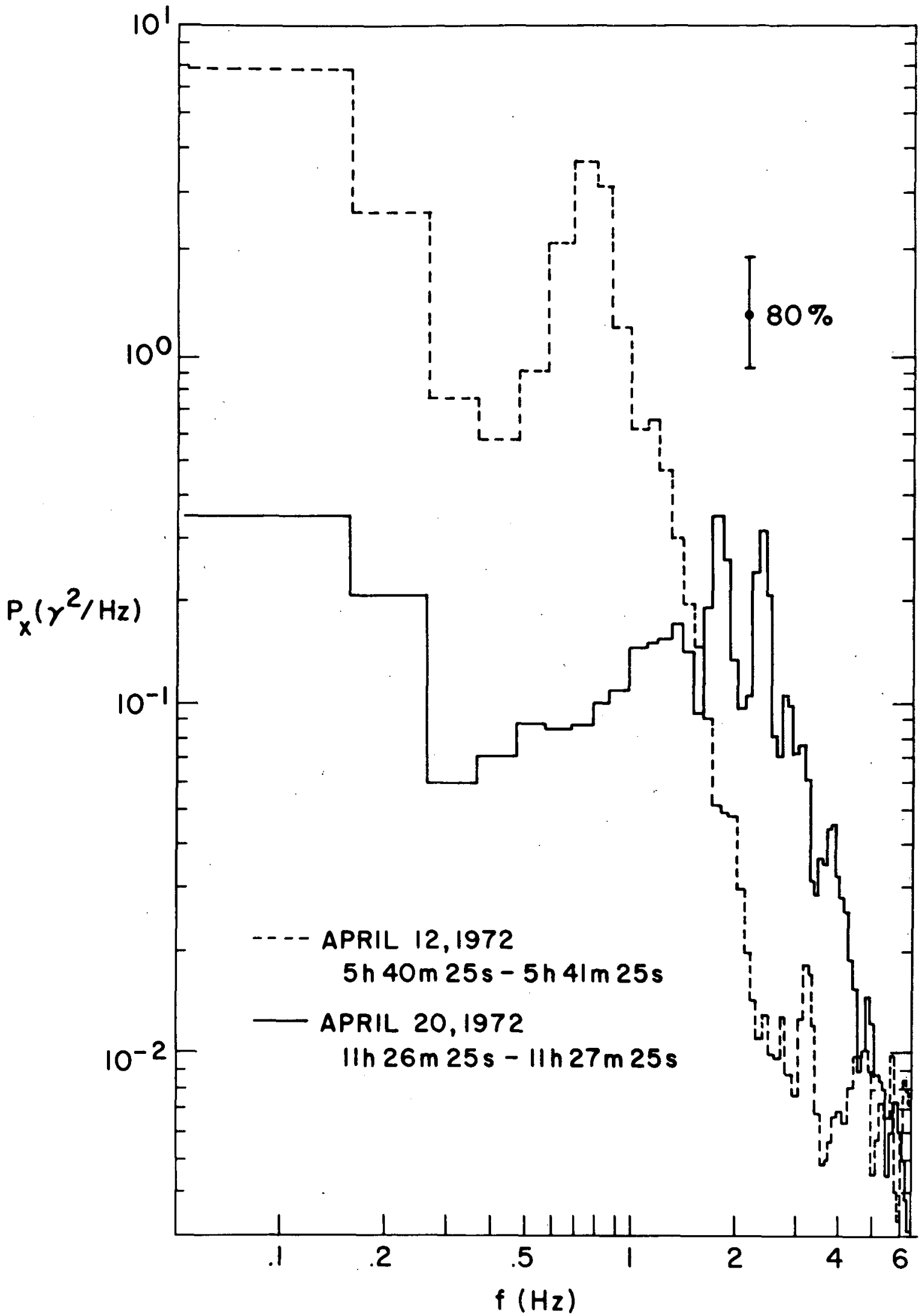

Figure 5 


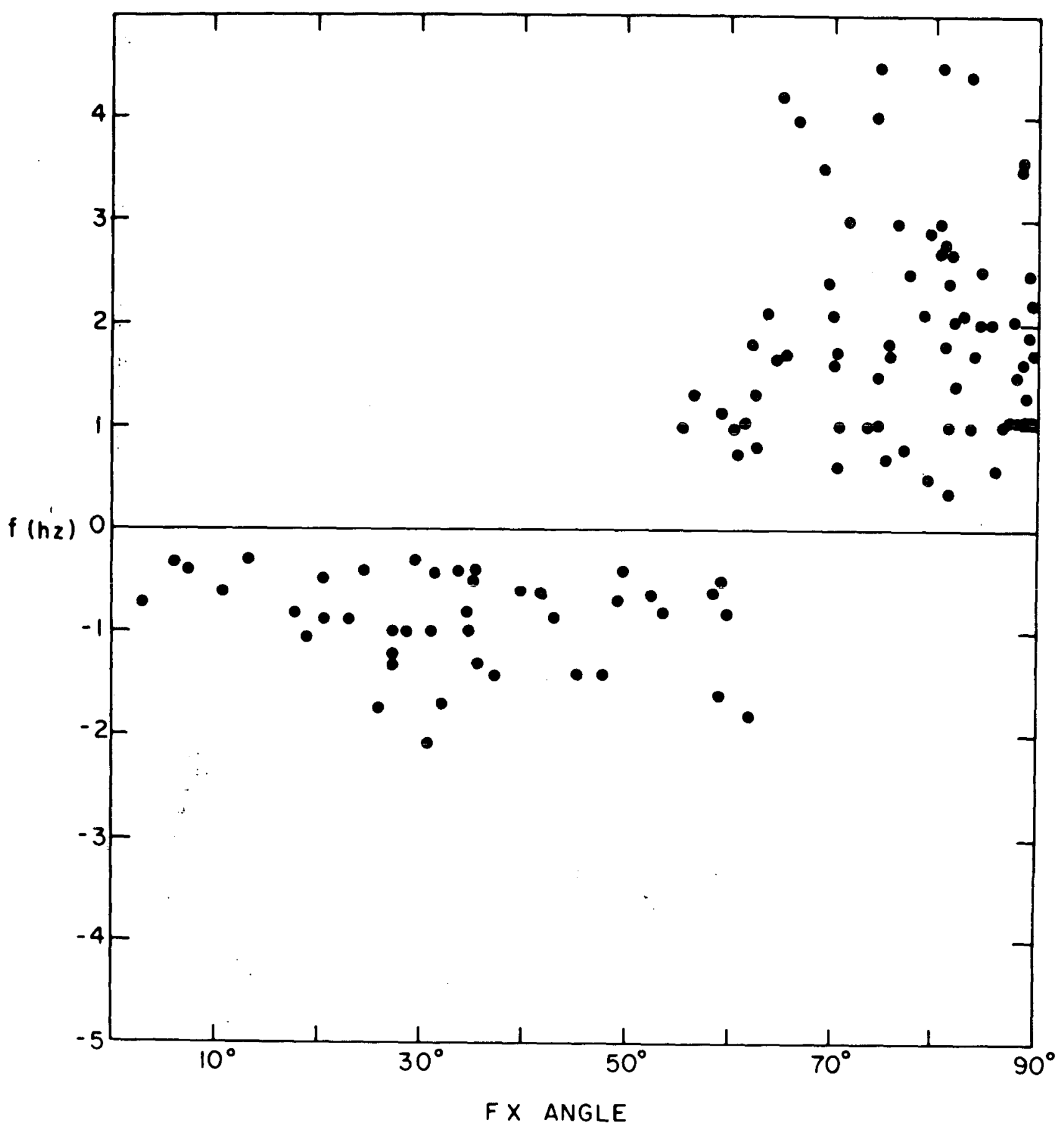

Figure 6 


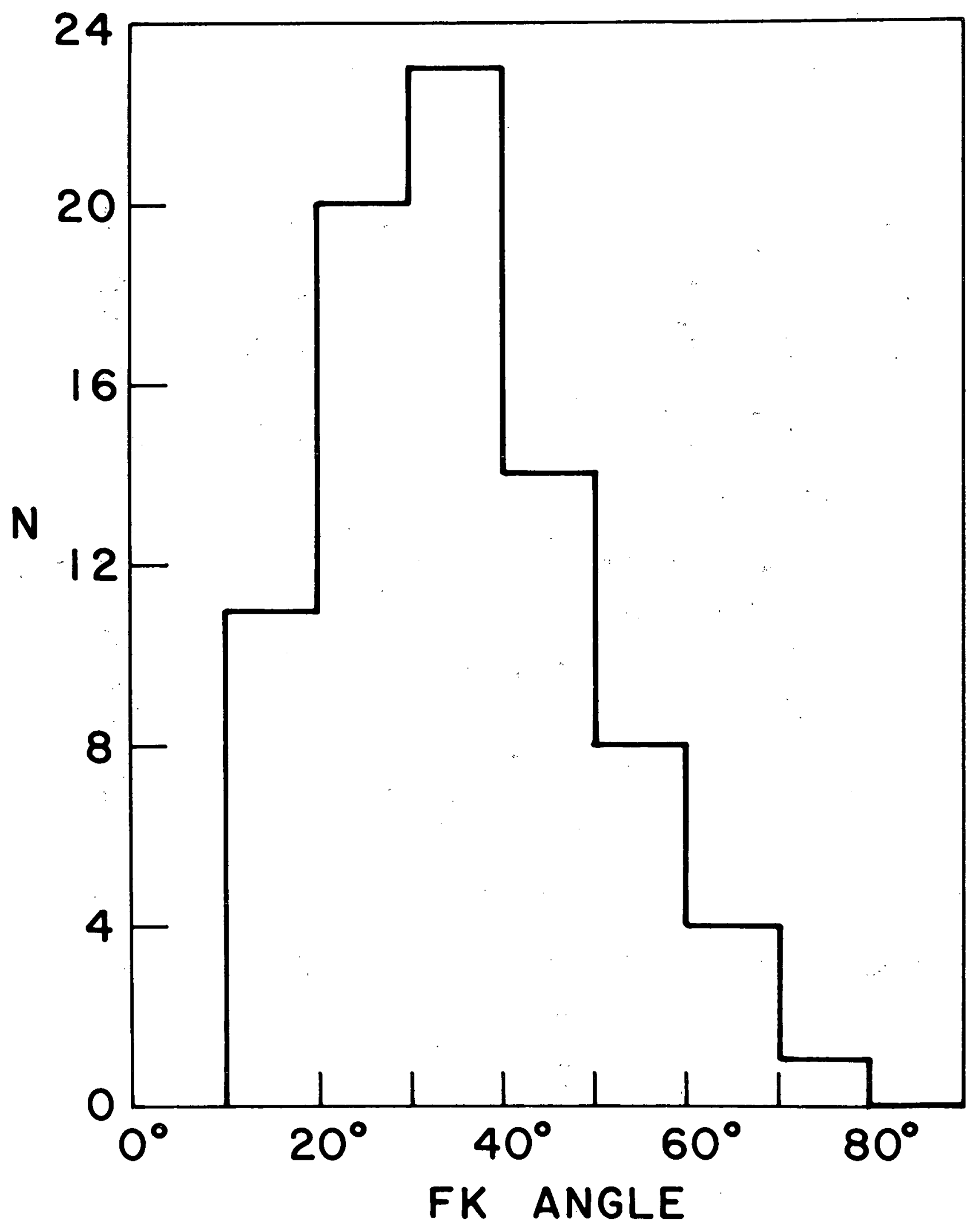

Figure 7 


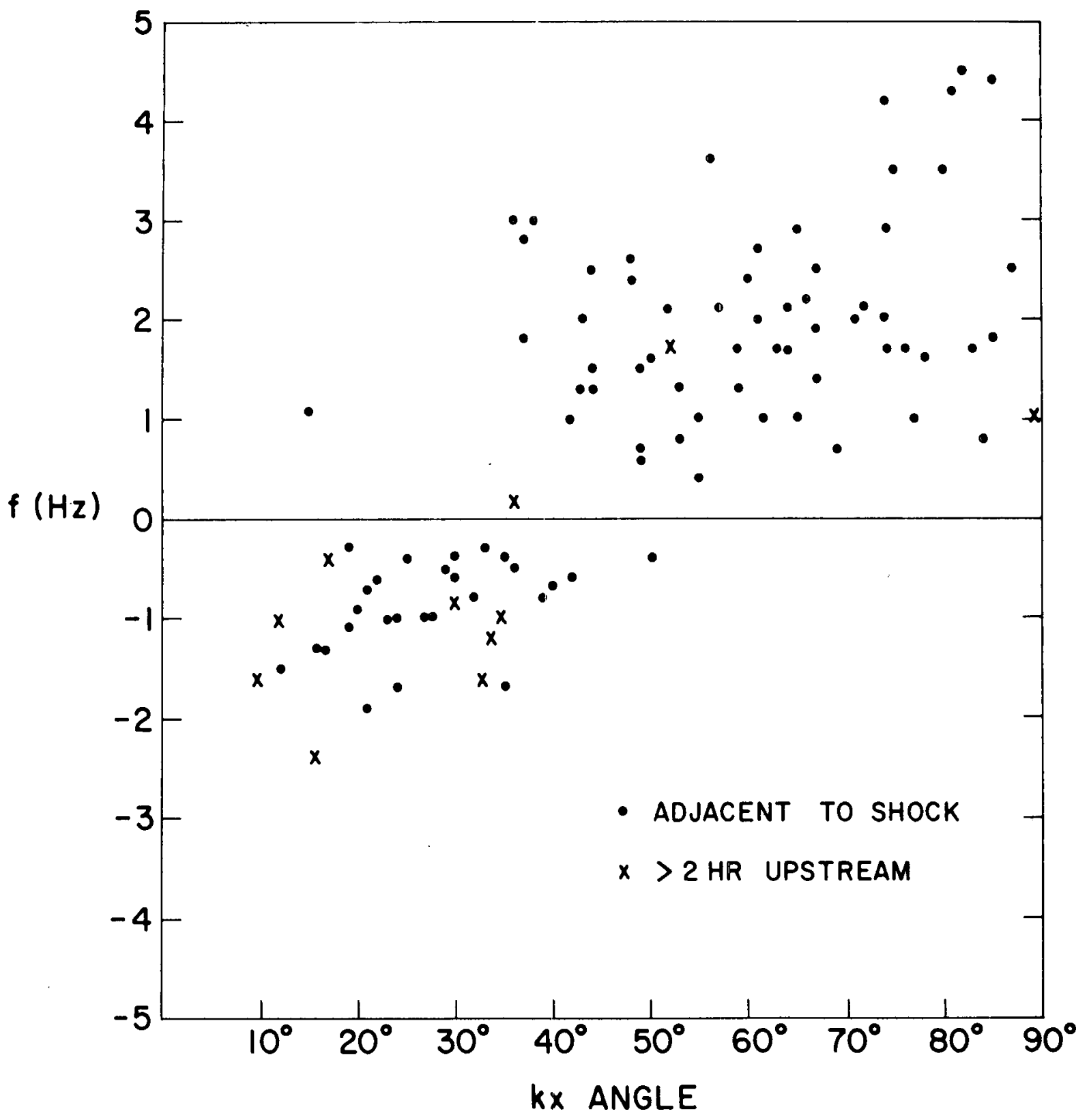

Figure 8 


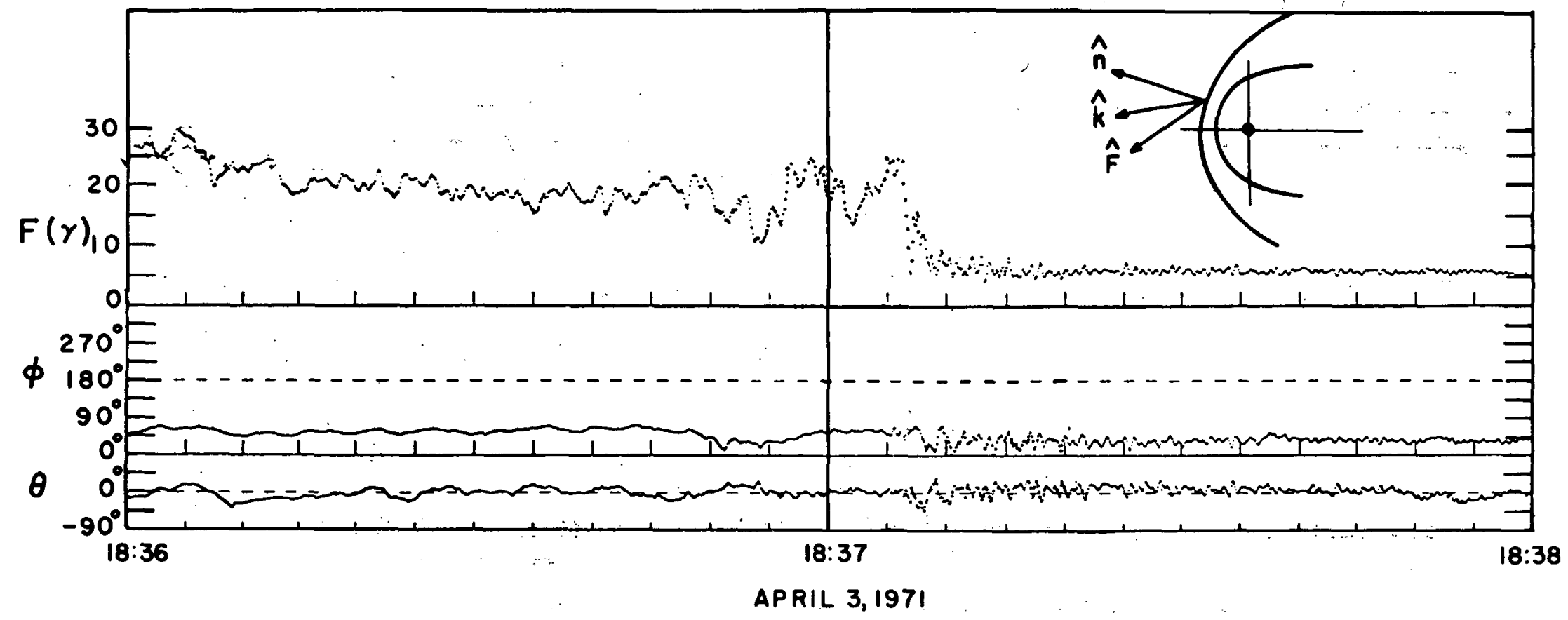

Figure 9 

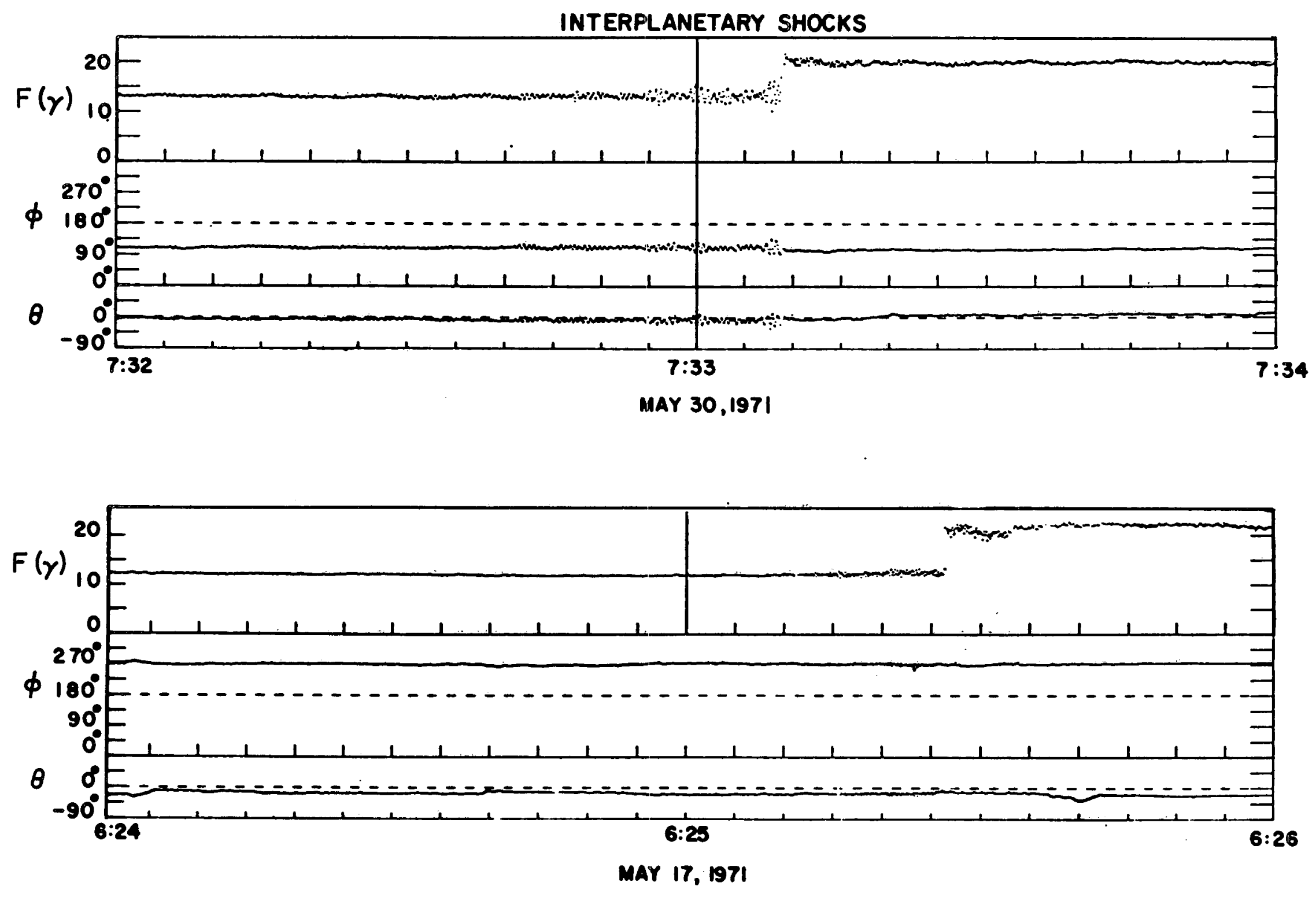

Figure 10 


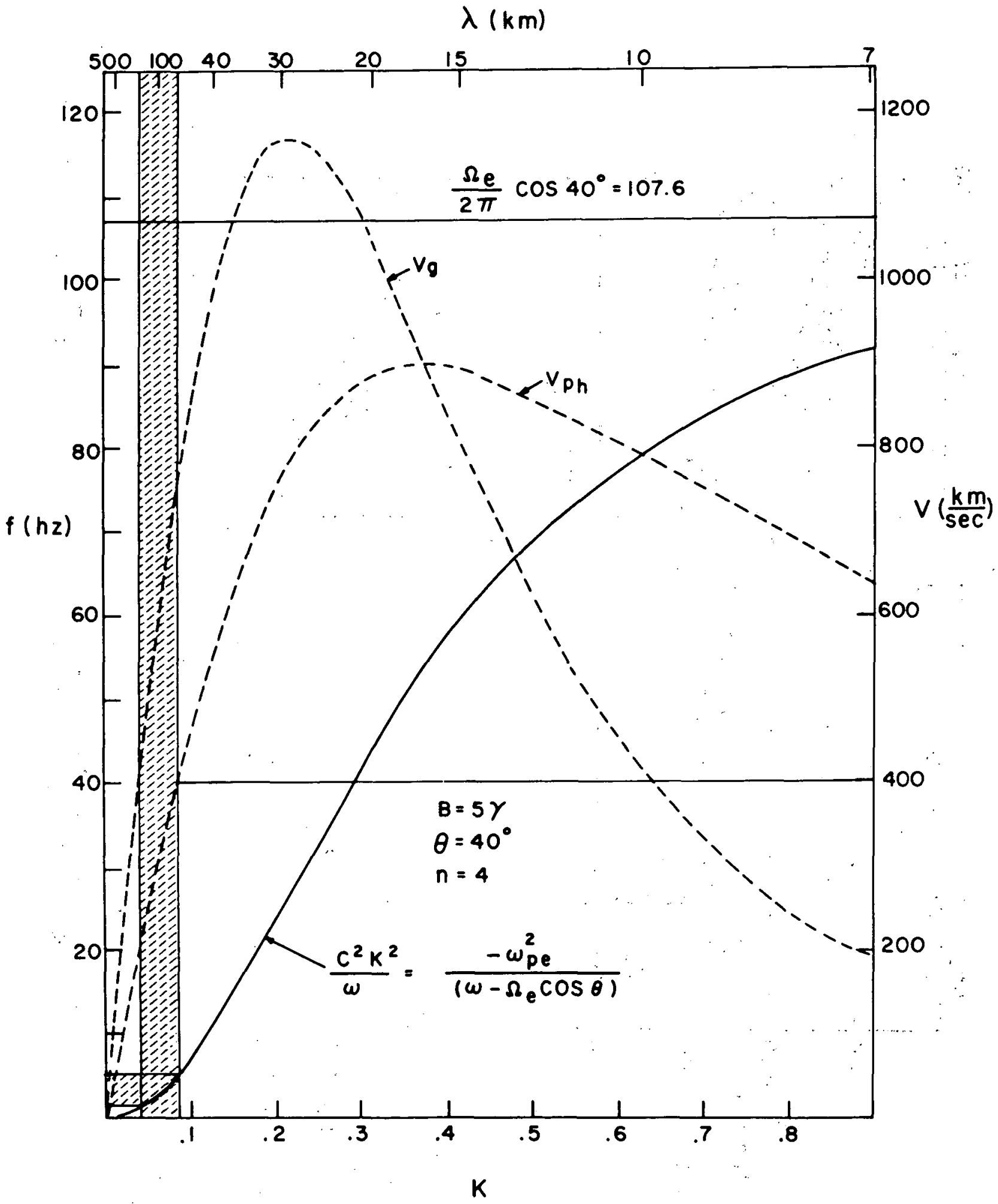

Figure 11 


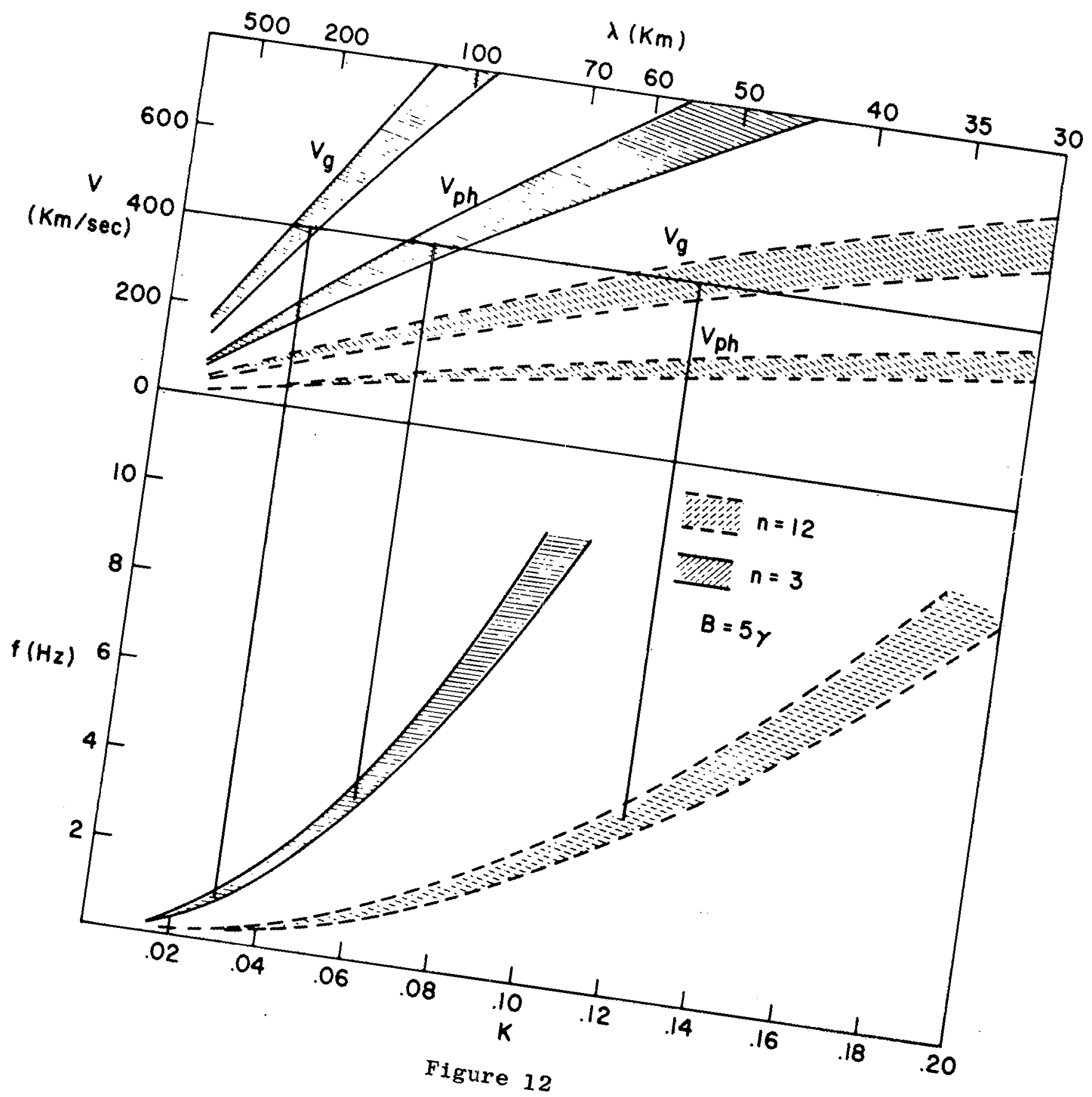

NASA-GSFC 